pop up window once you click on the image itself. It also features two links to enlarge and reduce the images, although these were not working during a visit to the site in December 2012.

The collection contains frontispieces and isolated folios and pages of medical, natural history and botanic treatises, as well as fragments of scientific reports, public health campaign materials, and photographs from archives and libraries in Spain, France, the United Kingdom and the United States. Also represented is a rich collection of artefacts related to medical treatments for malaria, the production and storage of quinine, and the identification and classification of the mosquito vector. The original materials are housed in museums in Madrid, Seville, Barcelona, as well as the Wellcome Library in London, and private collections. Highlights of the exhibit include images of eighteenthcentury bloodletting instruments including two beautiful bloodletting cups from Aragon. There are also pictures of plaster models used for bloodletting training involving leeches and lancets; eighteenth-century botanical cinchona samples from New Granada collected during the José Celestino Mutis scientific expedition in the eighteenth century; public health campaign posters from the 1920s in Spain; and a multicoloured array of medicinal product packaging from Spain and Italy.

The website certainly serves its main purpose as a general introductory catalogue to the themes and holdings of the BNE on the subject. The fact that the website is in Spanish limits its utility as an educational resource outside Spanish-speaking countries. At the same time, the striking images can be very useful in a classroom setting as part of lectures or workshops. Although it will disappoint specialists looking to access specific primary sources for their own research, the website does help in identifying initial points for exploring collections in less prominent Spanish archives. In summary, the 'Malaria' virtual exhibit is a concrete, well-organised, and beautifully designed general introduction to the subject. Even though it is in Spanish, it is readily accessible to educators in other languages and can be useful to historians working on the history of malaria during their first forays into Spanish archives.

Pablo F. Gómez The University of Wisconsin, USA

doi:10.1017/mdh.2013.2

\title{
The University of Virginia Claude Moore Health Science Library Online \\ Collections, available online at http://www.hsl.virginia.edu/historical/exhibits2.cfm
}

Scholars interested in early modern medicine will be rewarded by a visit to the website of the Claude Moore Health Sciences Library of the University of Virginia. For more than a decade, the staff of the Historical Collections and Services have been at work curating a series of digital exhibits that present and contextualise some of its resources. In some half a dozen exhibits focused on colonial North America and Europe, visitors are reminded of the creativity with which people coped with the precariousness of life in the pre-modern world.

Two exhibits are primarily catalogues of early editions of technical medical treatises owned by the Library. Vaulted Treasures offers sample pages from clinical and theoretical works from the sixteenth to nineteenth centuries, including path-breaking treatises on 
circulation and postmortem anatomies (William Harvey), herbal medicine (Nicholas Culpepper), and clinical study (Thomas Sydenham), along with links to the full text when available and brief introductions to each work. Twenty Medical Classics of the Jefferson Era is similarly rudimentary in its contextual apparatus, characterising Jefferson's time as one of clinical trials and advancements in anatomical, surgical, botanical and medical studies on both sides of the Atlantic. Links to the online texts would have been useful.

Both exhibits testify to the fact that we continue to imagine learned texts in isolation from other medical literature and practitioners - curious given what we know about professional and scientific medicine: that the dissemination of inoculation and vaccination relied heavily on the interactions of clerics and physicians with servants and enslaved Africans; that popular or 'vernacular' advice manuals translated even technical medical learning for a broader audience of laypeople (Harvey's observations on the blood's circulation made their way into several); that in London bookstores, Latin medical texts - perhaps the most learned and inaccessible to laypeople - shared shelf space with manuals and recipe books in English. In other words, these genres occupied a shared social context and cultural framework, which should warn against separating them for an online exhibit.

Fortunately, others showcase in more creative ways the intersection of early modern genres and classes of people. Bouts with plague spawned a genre of reporting, often called plague books, that chronicled social and spiritual responses across a range of institutions, including municipal councils, hospitals, brotherhoods and confraternities. The exhibit 'Orders thought meete ... ': Elizabethan Plague Book purports to be about one such book, but in fact features a set of seventeen regulations promulgated by Queen Elizabeth in 1578 and printed subsequently with advice from London's College of Physicians. The regulations, an attempt to formulate a standard emergency response, demonstrate the interest of early modern states in taxing, punishing, sanitising, feeding, medicating and pressing subjects into service as inspectors and enforcers of segregation orders, all in the interest of controlling disease and its social effects. While the source cannot divulge the extent to which the English state succeeded in controlling behaviours and enlisting members of society, the accompanying medical advice sheds light on common notions of the cleansing power of herbs - rosemary, lavender, sage, juniper - as well as vinegar and fire smoke, suggesting the centrality of the senses in defining and perceiving pestilence and purity.

Therapeutics receiving the endorsement of Elizabethan physicians proved durable and remained in common use centuries later in the Americas. Patients' Voices in Early Nineteenth Century Virginia features letters sent to James Carmichael and his two sons, physicians practising in Fredericksburg, Virginia, between 1819 and 1830. Helpfully transcribed for visitors, these letters, part of a 700-piece collection acquired by the University of Virginia in 1998, open a panoramic vista onto the expectations of patients in interaction with physicians. Authors request advice, medications, or house visits and often refer to quinine, mercury, cathartics, purges, caustics, bloodletting, opiates and 'good regimen' or 'diet'. Many are concerned with enslaved workers on plantations in need of care. Transcriptions of professional correspondence shed light on some more challenging medical mysteries: a colleague writes to Edward Carmichael for advice on a postmortem anatomy that revealed a 70-year-old woman to be 'half-man, half-woman'.

Amid numerous revelations is evidence that residents in Virginia were turning to vaccination, still a novelty in the decades following Jenner's 1798 publication, only when an epidemic of smallpox appeared imminent. A letter from Mary Skinker, dated 9 April 1828, read: 
We are in a great state of alarm, having heard this morning that our neighbour Mr Henry Somerville, has the small-pox.... We wish you to come up, and vaccinate our Children \& negroes.... I have had the small-pox, Mr S.r has been vaccinated, but the children did not take the vaccination, administered by you, when you were last here.

Why didn't they, if it was available? In the absence of state sanctions, the salience of an impending crisis - and attendant loss of life and profit - apparently proved effective in moving contemporaries to vaccinate dependents.

An exhibit curated for the bicentennial celebration for the departure of the Lewis and Clark Expedition centres non-Europeans in this history in other ways. Medicine \& Health on the Lewis and Clark Expedition reminds us of the expedition's ethnographic component: members took explicit interest in the healing, childrearing, and dietary habits and diseases of Native American communities, and colonial therapies and medications became important in other ways as members sought to forge good relations with groups encountered along the way through the healing arts. Curiously, Jefferson's distrust of the excesses of regular physicians led to the omission of a trained physician on the expedition; responsibility redounded to Lewis, who had been mentored by his herbalist mother and by the famed physician Benjamin Rush. Purgatives, laxatives, febrifuges, pain relievers, and herbal remedies were common, and members were apparently encouraged to implement Rush's 'Ten Rules' for good health. (Would members have steeped meat for a day or two in lye in the absence of salt (rule 5) or worn shoes without heels (rule 9)?) The expedition soon became a 'walking hospital', afflicted by fevers, dysentery, rheumatism, constipation, venereal disease, and malaria. Excerpts from Clark's journal lend vivid detail to the exhibit, while portraits of key personalities and images of remedies and tools from the library's collection provide ample context and a remarkable window onto contemporary notions of illness and health - an infrequently explored dimension of the famous United States expedition.

To great effect, this exhibit combines text and image, which were, as Ludmilla Jordanova, Roy Porter and Sander Gilman have shown, intimately intertwined in early modern medical literature. This was a highly visual culture, in which skeletal and anatomical drawings worked with textual explanations to illuminate new concepts, portraits of authors lent authority to medical texts, and contemptuous caricatures knocked physicians from their pedestals. Some marvellous examples of the popular uses of visual imagery appear in Very Ill! The Many Faces of Medical Caricature in Nineteenth Century England and France, which illustrates vividly the kinds of pressures a lay public might exert on medical practitioners. In one 1804 London etching, by the cartoonist James Gillray, the patient looks away from his arm, which gushes blood into a bowl, with something akin to a dejected frown; the physician, eyes wide and head inclined, seems overly keen to witness his patient's dismay. Other prints in this colourful collection take aim at the dupes of quack medicine or the overconsumption of the upper, middle and lower classes. An 1819 print by George Cruikshank, who illustrated some of Dickens's books, is titled simply Head Ache and depicts what appear to be goblins and imps variously drilling, shouting, poking and hammering at the head of a chair-bound sufferer. As critiques of greed, incompetence, hubris and excessive credulity, these images were satirical; as illustrations of the ways people suffered, succumbed to spurious knowledge masquerading as science, and paid dearly for the promise of relief - which often brought only more pain and suffering - they are sobering reminders that laughter, if not the best medicine, was sometimes the only medicine to be had. 
Together these exhibits, which highlight books, manuscripts, prints, and artefacts in the collection, demonstrate the possibility of conjuring a richer universe of healing when explicitly medical texts are joined with images and general works on the cultural, political, and ethnographic dimensions of particular times and places. While some exhibits require updating - nearly half of the links to related websites were broken - insofar as they have no closing date and can be accessed from the classroom or office, they are well worth the small price of admission.

Paul Ramírez

Northwestern University, USA 